\title{
An investigation into the adequacy and perception of benefits and barriers of physical activity in relation to the nutritional status of university students
}

Physical inactivity (PI) is the fourth leading risk factor for global mortality and contributes to an estimated $3 \cdot 2$ million deaths per year globally $^{(1)}$. At national level, PI costs the public sector an estimated $£ 940$ million per year ${ }^{(2)}$, while it also increases the risk of non-communicable diseases such as Coronary Heart Disease and Type 2 diabetes and adversely affecting the mental health ${ }^{(3)}$. Previous studies indicated that less than half of university students meet the guidelines for physical activity (PA) of 150 minutes of moderate to vigorous intensity PA per week ${ }^{(4)}$. The aim of the study was to investigate the adequacy of PA habits and the perception of PA benefits and barriers and to explore the differences in nutritional status of PA achievers and non-achievers.

After obtaining ethical approval, participants ( $n$ 234) aged between 18-41 from universities in the North West of England were recruited via convenience and snowball sampling in a cross-sectional study. Current PA and perception of the benefits and barriers of exercise were assessed via a validated physical activity diary and a validated exercise benefits/barriers scale (EBBS) questionnaire ${ }^{(3)}$ respectively. Self-reported levels of PA were then used to identify PA achievers (As) and non-achievers (NAs). The assessment of nutritional status included anthropometrical and physical measures such as height, weight, waist and hip circumferences, body composition and blood pressure as well as biomarkers of fasting capillary blood lipid profile. Normal distribution was assessed using the Shapiro-Wilk test of normality and because the main variables were not normally distributed $(\mathrm{P}<0.05)$, the difference between two groups was investigated using the Mann-Whitney $U$ Test. Statistical analysis was conducted using SPSS v22 and statistical significance was set at $0 \cdot 05$.

Findings revealed that $64 \%$ of students were meeting PA guidelines. The median minutes of moderate and vigorous PA in As were 330 per week in comparison with 60 per week in NAs. There was a significant difference in the EBBS score between the groups, suggesting that PA NAs had higher positive perception of PA, in spite of not achieving the guidelines. The majority of anthropometric and blood lipid parameters showed no significant differences between the groups with the exception of body fat, which was statistically higher in PA NAs.

\begin{tabular}{|c|c|c|c|c|c|c|}
\hline \multirow[b]{3}{*}{ Parameters } & \multicolumn{3}{|c|}{ PA achievers (n 152) } & \multicolumn{3}{|c|}{ PA non-achievers ( $n$ 82) } \\
\hline & \multirow[b]{2}{*}{ Median } & \multicolumn{2}{|c|}{ Quartiles } & \multirow[b]{2}{*}{ Median } & \multicolumn{2}{|c|}{ Quartiles } \\
\hline & & $\mathrm{P} 25$ & P75 & & $\overline{\mathrm{P} 25}$ & P75 \\
\hline PA minutes per week & $330 *$ & $240 \cdot 0$ & $480 \cdot 0$ & $60 \cdot 0 *$ & $0 \cdot 0$ & $120 \cdot 0$ \\
\hline EBBS score & $78 *$ & $67 \cdot 0$ & $87 \cdot 0$ & $86 \cdot 5 *$ & $76 \cdot 0$ & $96 \cdot 0$ \\
\hline PA barriers score & $43 *$ & $39 \cdot 3$ & $47 \cdot 0$ & $40 \cdot 5 *$ & $36 \cdot 0$ & $44 \cdot 3$ \\
\hline PA benefits score & $49 *$ & $41 \cdot 0$ & $57 \cdot 0$ & $56 \cdot 0 *$ & $49 \cdot 0$ & $63 \cdot 0$ \\
\hline BMI $\left(\mathrm{Kg} / \mathrm{m}^{2}\right)$ & $23 \cdot 7$ & $21 \cdot 5$ & $25 \cdot 6$ & $23 \cdot 8$ & $20 \cdot 7$ & $27 \cdot 3$ \\
\hline Body fat $(\%)$ & $22 \cdot 5^{*}$ & $16 \cdot 3$ & $29 \cdot 9$ & $27 \cdot 6^{*}$ & $21 \cdot 0$ & $34 \cdot 0$ \\
\hline Waist to hip ratio $(\mathrm{cm})$ & $0 \cdot 8$ & $0 \cdot 7$ & $0 \cdot 9$ & $0 \cdot 8$ & $0 \cdot 7$ & $0 \cdot 8$ \\
\hline Total cholesterol (mmol/L) & $4 \cdot 1$ & $3 \cdot 5$ & $4 \cdot 5$ & $4 \cdot 0$ & $3 \cdot 4$ & $4 \cdot 5$ \\
\hline Total HDL (mmol/L) & $1 \cdot 3$ & $1 \cdot 1$ & 1.6 & 1.4 & $1 \cdot 2$ & $1 \cdot 6$ \\
\hline Total LDL (mmol/L) & $2 \cdot 1$ & $1 \cdot 6$ & $2 \cdot 6$ & $2 \cdot 2$ & $1 \cdot 8$ & $2 \cdot 5$ \\
\hline
\end{tabular}

Key: * represent significant $(\mathrm{P}<0.05)$ differences between the groups

In conclusion, the present study demonstrated that while more than a third of university students do not meet the PA recommendations, the underlying explanation for this might not be the perception of the benefits of PA or its barriers. The future research should include a larger sample size, with further consideration of the socioeconomic and gender variations to further characterise the anthropometric and nutritional status profile of the PA As and NAs.

1. World Health Organisation (2015) Physical activity [Online] http://www.who.int/topics/physical_activity/en/

2. Pratt, Norris, Lobelo et al. (2012) Br J Sports Med 48, 1627-1633.

3. Warburton, Nicol, Bredin (2006) CMAJ 174, 801-809.

4. Irwin (2004) Percept Mot Skills 98, 927-943. 